\title{
A Study of Gallium FIB induced Silicon Amorphization using TEM, APT and BCA Simulation
}

Jin Huang ${ }^{1}$, Markus Loeffler ${ }^{1}$, Uwe Muehle ${ }^{2}$, Wolfhard Moeller ${ }^{3}$, Hans Mulders ${ }^{4}$, Laurens Kwakman ${ }^{4}$ and Ehrenfried Zschech ${ }^{1,2}$

1. Dresden Center for Nanoanalysis, Technische Universitaet Dresden, Dresden, Germany.

2. Fraunhofer Institute for Ceramic Technologies and Systems, Dresden, Germany.

3. Helmholtz-Zentrum Dresden-Rossendorf, Dresden, Germany.

4. FEI Company, Eindhoven, the Netherlands.

Crystalline silicon (c-Si) is partially amorphized in Focused Ion Beam (FIB) TEM lamella preparation. A $30 \mathrm{kV} \mathrm{Ga}^{+}$beam with a small glancing incident results in a $20-30 \mathrm{~nm}$ amorphous layer $[1,2]$. The precise mechanisms remain uncertain and a damage prediction can hardly be made. In this study, a Binary Collision Approximation (BCA) software is employed to simulate c-Si amorphization in various $\mathrm{Ga}^{+}-\mathrm{FIB}$ conditions. These results are compared with experimental data from transmission electron microscopy (TEM) and atom probe tomography (APT) of Si samples prepared by a FEI Helios 660 FIB/SEM system.

BCA simulation is widely used for ion-solid interactions, however, simulation of the FIB processes requires taking dynamic phenomena into account. The TRIDYN code treats the deceleration of ions in solids and the associated formation of recoil atom cascades in the BCA model [3]. In contrast to the TRIM code [4], it considers dynamic alterations of the local composition which arise from the ion implantation process. In this way, phenomena such as sputter erosion, atomic mixing and recoil implantation can be simulated with improved accuracy. Due to the limitation of the BCA model, TRIDYN is unable to simulate any crystalline-to-amorphous transition. It was demonstrated by Monte Carlo simulation and experimental studies that $\mathrm{Si}$ amorphization can be described by average displacement of host-atoms, where the critical displacement is $5 \AA$ [5]. It was also shown that, in terms of atom displacement, the Molecular Dynamics method is more accurate, but does not have a significant difference to BCA modeling for $>5 \AA$ atom displacement [6]. In this context, TRIDYN can be employed for the simulation of Si amorphization during FIB under the mentioned assumptions. Figure 1 shows the amorphous layer thickness as a function of the $\mathrm{Ga}+$ ion energy at 3 different ion impingement angles: $3^{\circ}, 5^{\circ}$ and $7^{\circ}$. These curves behave differently in lower and higher $\mathrm{Ga}^{+}$energy ranges, which may indicate that the mechanisms are different.

TEM images of c-Si cross-sections after $\mathrm{Ga}^{+} \mathrm{FIB}$ preparation at 5 different ion energies are shown in Figure 2(a)-(e). C-Si samples are prepared as wedge lamellae using a standard $30 \mathrm{kV} \mathrm{Ga}^{+} \mathrm{FIB}_{\text {preparation }}$ method. Subsequently, a $\mathrm{Ga}^{+}$beam of $0.5,1,2,5$ and $30 \mathrm{kV}$, with a $5^{\circ}$ glancing angle is applied to the side of the wedge. Typically, when removing material, the energy-dependent damage and implantation layer that extends into the sample as material is removed. The experiment was designed in such a way that sufficient amorphous and crystalline material is removed to reach a steady state and to retain only an amorphous layer of this energy-dependent thickness. For comparison, the measured values are plotted in Figure 1. All experimental data correlate well with the model-based predictions, showing that TRIDYN is a valid and well adapted model to estimate amorphous layer thicknesses. Implanted Ga ion distributions have been modelled with TRIDYN and experimentally measured with APT as well: for this, c-Si samples are prepared with a specific cone geometry for APT measurements. In the last step of the preparation, a 5 $\mathrm{kV} \mathrm{Ga}+$ beam is applied collinearly to the tip direction, which results in a final cone angle of $\sim 22^{\circ}$. The $\mathrm{Ga}$ concentration along a radius on the axial cross-section is plotted in Figure 3. Note that the strong scatter 
at the shallow surface is caused by the insufficient number of detected atoms. A TRIDYN simulation of the $\mathrm{Ga}$ distribution with the same beam conditions is also shown for comparison. Although the exact relationship between $\mathrm{Ga}$ concentration and $\mathrm{Si}$ amorphization is not yet established, the highly matched data suggests TRIDYN can be considered as a reliable reference in future studies.

It is concluded that the FIB milling process induced damage and implant can be well described with the TRIDYN simulation. Optimized recipes for ultra-thin lamella preparation can be now engineered using this simulation model. For such critical lamellae, multiple ion energies have to be used, each for an optimized time to achieve the final lamella thickness in shortest time and with the minimum damage.

References:

[1] HJ Engelmann et al, Microscopy Today 11 (2003), 22-24.

[2] LA Giannuzzi, R Geurts and J Ringnalda, Microsc Microanal 11 (2005), 828-829.

[3] W Moeller, W Eckstein and JP Biersack, Computer Physics Communications 51 (1988), 355-368.

[4] JP Biersack and LG Haggmark, Nuclear Instruments and Methods 174 (1980), 257-269.

[5] LJ Gracia et at, Journal of Applied Physics 109 (2011), 123507.

[6] L Bukonte et al, Nuclear Instruments and Methods in Physics Research B 297 (2013), 23-28.

[7] The authors acknowledge the project funding from FEI and Makizu project funding from BMBF.
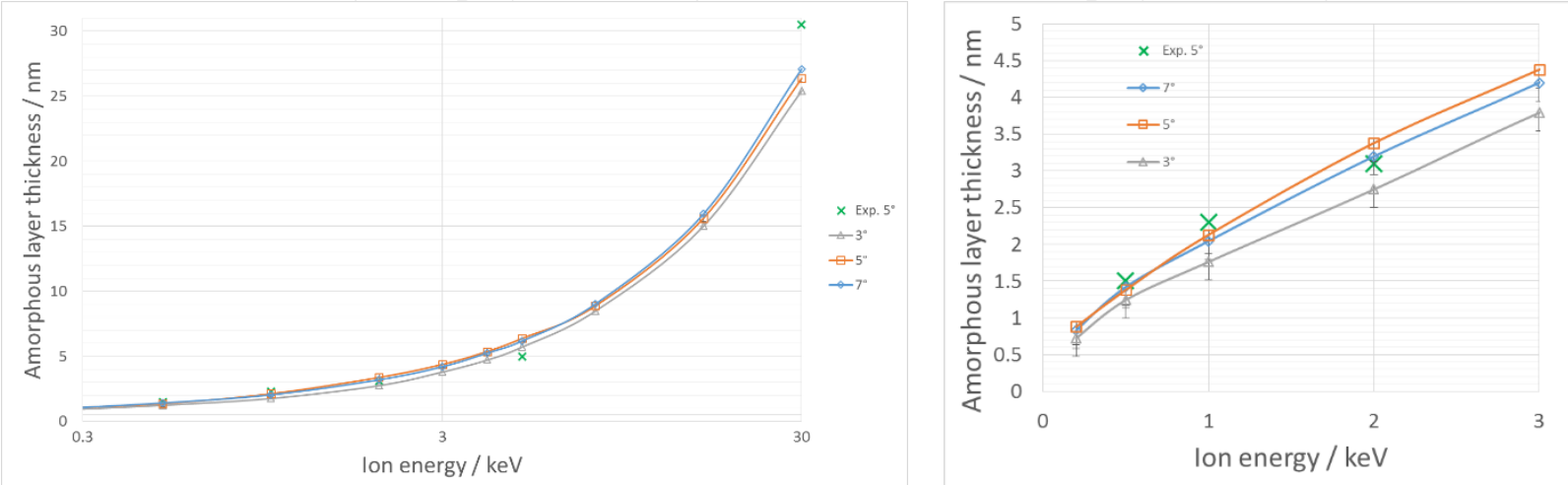

Figure 1. TRIDYN Simulated amorphous Si layer thickness as a function of ion energy for $3^{\circ}, 5^{\circ}$ and $7^{\circ}$ ion impingement angle. Experimental TEM data are also depicted for comparison.

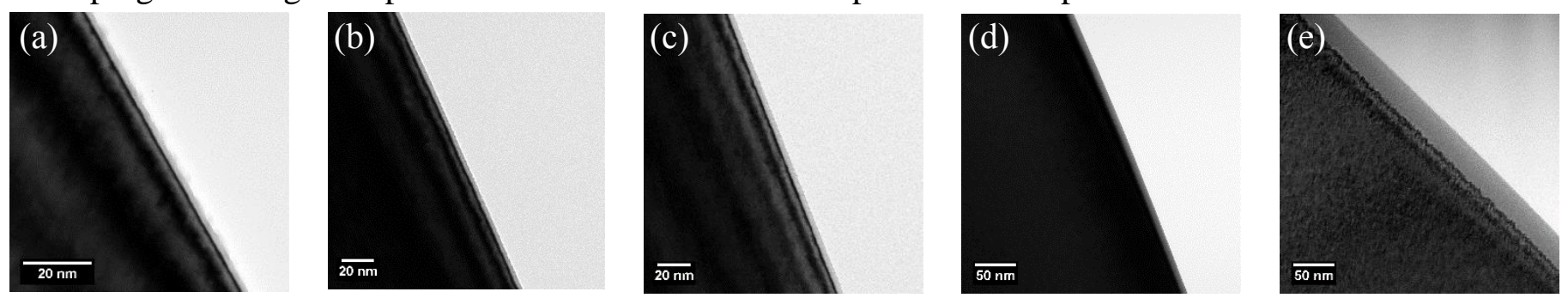

Figure 2. TEM images of a c-Si cross-section after a 0.5/1/2/5/30kV Ga FIB process show amorphous layer thicknesses of 1.5/2.3/3.1/5.0/30.5nm, from (a) to (e) respectively.
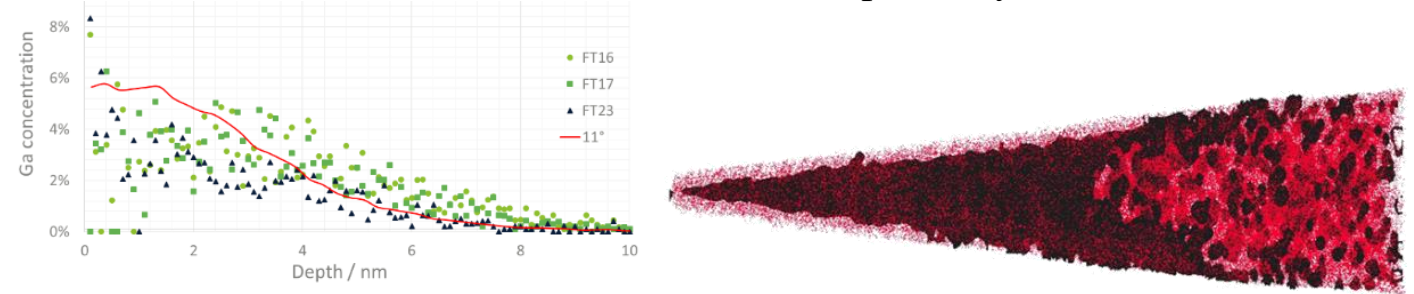

Figure 3. APT determined Ga concentration profiles from three APT samples, with corresponding TRIDYN simulation (left); illustration of APT measurement (Ga: black and Si: red) (right). 